\title{
Avaliação de usabilidade do Sistema Tutor Inteligente PAT2Math
}

\author{
Felipe de Morais, PIPCA - UNISINOS \\ felipedemoraisfm@hotmail.com \\ Patrícia Jaques, PIPCA - UNISINOS \\ pjaques@unisinos.br
}

\begin{abstract}
Resumo. A interface gráfica de um Sistema Tutor Inteligente é o principal meio de comunicação do usuário com o sistema. Porém, para uma interface ser considerada intuitiva e fácil de usar, ela deve ser submetida por um processo de avaliação de usabilidade. Este artigo descreve uma avaliação de usabilidade na interface gráfica do Sistema Tutor Inteligente PAT2Math. Para essa avaliação foi utilizado o método de avaliação heurística, o qual apontou várias limitações da interface em relação à sua usabilidade. Como solução para os problemas de usabilidade evidenciados foi desenvolvida uma nova interface gráfica baseada na tecnologia HTML 5.
\end{abstract}

Palavras-chave: Sistema Tutor Inteligente, Avaliação de Usabilidade, Interface Gráfica, Heurísticas de Usabilidade.

\section{Usablity Evaluation of the Intelligent Tutoring System PAT2Math}

\begin{abstract}
The graphical interface of an Intelligent Tutoring System is the main communication form between the user and the system. However, in order to an interface to be considered easy to use and intuitive, it must undergo an usability evaluation process. This paper describes an usability evaluation of the graphical interface of the Intelligent Tutoring System PAT2Math. We used the heuristic evaluation method, which showed that the interface had many usability drawbacks. As a solution to these problems, it was developed a new graphical interface based on HTML 5 technology.
\end{abstract}

Keywords: Intelligent Tutoring Systems, Usability Evaluation, Graphical User Interface, Usability Heuristics.

\section{Introdução}

Sistemas Tutores Inteligentes (STI) são softwares que tem como objetivo auxiliar os alunos no processo de ensino-aprendizagem. Estes softwares utilizam técnicas de Inteligência Artificial (IA) para fornecer assistência individualizada ao aluno (Koedinger et al., 1997). Esses sistemas vêm provando serem eficientes pedagogicamente. Segundo Woolf (2008), alunos que tem um auxílio individual de um tutor (relação professor/aluno 1:1), conseguem realizar $98 \%$ de suas tarefas, enquanto que alunos em um ensino tradicional (relação professor/aluno 1:30), realizam apenas $50 \%$ dessas mesmas tarefas.

Neste contexto, o PAT2Math (Personal Affective Tutor to Math) é um STI voltado ao ensino de álgebra elementar a alunos do ensino fundamental. PAT2Math possui um sistema especialista, baseado em regras de produção, que representa o 
conhecimento necessário para corrigir cada passo da resolução de uma equação algébrica, fornecendo feedback imediato ao aprendiz. O STI é também composto pelo PATequation, um editor inteligente, no qual o aluno pode resolver uma determinada equação da maneira que lhe for mais adequada, recebendo correções a cada resposta. É através dele que o aluno interage com o sistema, ou seja, através de sua interface gráfica o aluno pode resolver as equações e ao mesmo tempo receber o feedback de sua ação (Jaques et al. 2013; Seffrin et al. 2010).

A área de Interação Humano-Computador (IHC) envolve o estudo da maneira mais eficiente de interação de pessoas com computadores. Para tanto, busca-se avaliar a usabilidade de interfaces gráficas (GUI - Graphical User Interface), pois elas são o principal meio de comunicação entre o usuário e o programa computacional. Usabilidade pode ser definida como a aceitabilidade do usuário para com o sistema, permitindo a execução de tarefas de forma segura, simples, clara e efetiva (Jacko and Stephanidis, 2003).

Através de regras de usabilidade, os sistemas são submetidos a avaliações de usabilidade para comprovar a eficiência de sua interface. Algumas das regras de usabilidade mais conhecidas são as Heurísticas de usabilidade, definidas por (Nielsen, 1994), e as Regras de Ouro, definidas por (Shneiderman and Ben, 2003).

Neste trabalho, é descrita uma avaliação de usabilidade no Sistema Tutor Inteligente PAT2Math utilizando um método conhecido como Avaliação Heurística. Neste método, durante a realização de uma tarefa utilizando o sistema, um especialista deve verificar se a interface segue as heurísticas de usabilidade definidas por (Nielsen, 1994), sendo que, para qualquer inconformidade, o avaliador deve descrever quando e onde ela ocorre. Este tipo de método é bastante utilizado para avaliar software, pois detecta inúmeras irregularidades antes que o sistema seja submetido a uma avaliação de usabilidade com usuários potenciais do sistema.

Este artigo está organizado da seguinte forma. A Seção 2 descreve o STI PAT2Math e uma de suas ferramentas denominada PATequation. Na Seção 3 é apresentada a descrição das dez heurísticas de usabilidade. Na Seção 4 são detalhados alguns dos principais problemas encontrados na interface durante avaliações anteriores. A Seção 5 apresenta os resultados obtidos na avaliação da interface gráfica do sistema. Fechando este artigo, a Seção 6 descreve as conclusões finais e trabalhos futuros.

\section{PAT2Math}

PAT2Math é um Sistema Tutor Inteligente web para auxiliar o processo de ensinoaprendizagem de álgebra elementar a alunos do ensino fundamental. Além disso, como trabalho futuro, o sistema visa também levar em consideração as emoções do aluno através das suas ações na interface e das expressões faciais do aluno (Jaques et al. 2011), proporcionando uma experiência mais interessante e menos frustrante.

A arquitetura do sistema é dividida nos seguintes componentes, seguindo a arquitetura de (Psotka et al., 1988). A Base de domínio representa o conhecimento procedural (implementado como um sistema especialista) e declarativo do tutor. $\mathrm{O}$ Modelo do aluno contém as informações referentes ao conhecimento do aluno, representando o aluno no sistema. O módulo Tutor, baseado nas informações armazenadas no Modelo do aluno, aplica estratégias pedagógicas para a exibição dos 
conteúdos ao aluno. A Interface gráfica é o componente responsável pela comunicação do aluno com o sistema.

PATequation é uma das principais ferramentas do PAT2Math. Ele é um editor inteligente de equações algébricas, na qual o aluno pode resolver uma determinada equação passo-a-passo, da maneira que mais lhe for conveniente. Para cada passo da resolução de uma equação fornecida pelo aluno, a interface do PATequation é responsável por fornecer feedback imediato. Caso o aluno tenha alguma dúvida ou dificuldade em algum determinado passo, ele pode pedir uma ajuda ao sistema, que lhe fornece uma dica inteligente (ver (Seffrin et al. 2012) para mais detalhes). O sistema também fornece dicas específicas quando o aprendiz comete algum erro em qualquer parte da resolução de uma equação.

Pat é um Agente Pedagógico Animado, incorporado ao PATequation, que tem como objetivo auxiliar os alunos na resolução das equações. Juntamente com a interface gráfica, Pat é responsável por fornecer dicas e feedbacks através de mensagens e comportamentos físicos. Isso proporciona uma maior interatividade ao aluno, visando evitar que o mesmo fique frustrado ao usar o sistema.

Este artigo tem como objetivo avaliar a usabilidade da interface gráfica do PATequation. Para isso, é utilizada uma avaliação heurística, com avaliadores especializados em desenvolvimento web. O principal objetivo é identificar e corrigir os principais problemas de usabilidade, de modo que a interface gráfica complemente a eficiência do sistema como um todo.

\section{Heurísticas de Usabilidade}

A fim de permitir que os alunos utilizem o sistema de forma simples e eficiente, a interface gráfica do PATequation segue as heurísticas de usabilidade propostas por (Nielsen, 1994). Essa escolha é devido ao fato de que as regras cobrem muitas das possíveis iterações do aluno com o sistema. As dez heurísticas de usabilidade são descritas abaixo:

Heurística 1: Visibilidade do estado do sistema. O sistema deve apresentar informações suficientes sobre seu estado, dando um feedback imediato para cada ação do usuário. Isso evita que o usuário fique frustrado ou confuso com o sistema. Por exemplo: ao verificar a resposta de uma equação o sistema deve informar se a mesma está correta ou não por meio de mensagens ou através de ícones.

Heurística 2: Mapeamento entre o sistema e o mundo real. Termos específicos de sistema devem ser evitados, pois alguns usuários podem não estar familiarizados com eles. Deve-se utilizar uma linguagem natural, clara e concisa. Outro aspecto interessante é sobre o mapeamento de aspectos do mundo real, utilizando o conhecimento que o usuário já tem. Por exemplo, as operações algébricas do sistema podem ser representadas da mesma maneira que uma calculadora normal.

Heurística 3: Liberdade e controle do usuário. O usuário deve se sentir no controle do sistema. Caso ele tenha feito algo errado, ele pode desfazer ou ainda refazer sua ação, dependendo do contexto. Por exemplo, caso o usuário deseje visualizar resoluções anteriores, ele pode selecionar a equação desejada, visualiza-la e voltar para a resolução da equação atual.

Heurística 4: Consistência e padrões. Ações similares e seus respectivos feedbacks devem seguir um padrão. Quanto à consistência, o layout e o posicionamento 
de itens na interface também devem seguir uma terminologia padrão, o que permite uma melhor visualização e contextualização do usuário.

Heurística 5: Prevenção de erros. As interfaces de usuário devem ser projetadas de modo que a ocorrência de erros seja mínima. A simplicidade das telas evita que o sistema se torne complexo, evitando possíveis falhas de compreensão do usuário.

Heurística 6: Minimizar a carga de memória - reconhecer em vez de relembrar. É importante que o fluxo de utilização do sistema seja simples, evitando que o usuário tenha que decorar processos de execução. O usuário deve reconhecer esses processos para não armazenar em sua memória de trabalho informações inúteis. Baseado em princípios psicológicos, a sobrecarga na memória de trabalho pode prejudicar a aprendizagem do aluno.

Heurística 7: Flexibilidade e eficiência de uso. Embora seja complexo, as interfaces deveriam ser flexíveis e customizáveis para cada usuário, aumentando assim sua eficiência. O sistema deve ser de fácil compreensão para usuários leigos. Entretanto, para usuários mais avançados deve haver suporte a atalhos e simplificações, fornecendo métodos mais ágeis para determinadas ações.

Heurística 8: Design estético e minimalista. A simplicidade no design da interface, na maioria das vezes, obtém melhores resultados de usabilidade. O sistema deve exibir somente o necessário, pois uma grande quantidade de opções pode confundir o usuário.

Heurística 9: Suporte para o usuário reconhecer, diagnosticar e recuperar erros. Caso o usuário cometa um erro, o sistema deve prover ajuda suficiente para informa-lo sobre o erro. Se possível informar também uma possível solução para o erro encontrado. Não deve ser utilizando mensagens com uma linguagem muito específica, como, por exemplo, "404 not found".

Heurística 10: Ajuda e documentação. A fim de ajudar o usuário, o sistema deve prover um sistema de ajuda e uma documentação técnica do funcionamento, quando isso for solicitado pelo usuário. Porém, o design da interface deve evitar que o usuário necessite algum tipo de ajuda. A ajuda deve ser facilmente acessada pelo usuário. Também deve ser de fácil navegação, permitindo pesquisas a um determinado tópico.

\section{Avaliações anteriores do PAT2Math}

Avaliações anteriores do sistema também foram importantes para o desenvolvimento e melhoramento da interface gráfica. Essas avaliações não tinham o objetivo de avaliar a interface gráfica, mas sim outros aspectos do sistema, tais como aprendizagem proporcionada, sistema de dicas, etc (ver (Jaques et al. 2013; Seffrin et al. 2010, 2012, 2009) para mais detalhes).

Cada vez que os alunos utilizavam o sistema, em alguma destas avaliações, e se deparavam com algum problema, eles pediam ajuda ao responsável pela avaliação, que por sua vez, relatava os problemas e sugestões dos alunos na utilização do sistema aos desenvolvedores do projeto. Ao avaliar esses problemas e sugestões, foi gerada uma lista de inconformidades, que auxiliou a equipe a identificar problemas na interface e correções a serem realizadas. Entre elas, podem-se destacar as seguintes:

Não desfazer ações indesejáveis: ao resolver uma equação, caso o usuário digitasse algum valor errado, ele deveria clicar em um botão simbolizando um apagador 
e então apagar o valor, o que não era intuitivo. A solução encontrada foi adicionar botões de desfazer e refazer. Sendo assim, para qualquer erro do usuário, agora ele pode facilmente desfazer ou refazer sua ação.

Clicar no botão de verificação: para verificar um passo na resolução de uma equação, o usuário deveria, obrigatoriamente, clicar em um botão com um símbolo de uma lupa. Porém, para usuários mais avançados, esse processo se tornava incômodo. A solução para este problema foi adicionar um atalho no teclado. Agora o usuário pode entrar com um passo da resolução e simplesmente pressionar a tecla "Enter", obtendo feedback imediato.

Barra de rolagem no quadro: a interface exibe a resolução das equações em um painel no formato de um quadro negro. Porém, ao adicionar novos passos na resolução de uma equação, o usuário deveria mover a barra de rolagem do quadro manualmente. Este processo também ia se tornando incômodo, além de aumentar o tempo na resolução de uma equação. Para solucionar este problema, cada vez que o usuário verifica um passo da resolução, o sistema recalcula automaticamente a posição da barra de rolagem, mantendo a última linha sempre no centro do quadro.

Botões das operações misturados: para cada operação algébrica, utilizada na resolução das equações, existe um botão na interface. Esses botões eram apresentados ao usuário sem nenhuma diferenciação entre eles, misturando os botões de operações de equações de primeiro grau com os de segundo grau, e ainda com os botões das ferramentas da interface. A solução encontrada foi adicionar quadros específicos para cada tipo de funcionalidade. Agora o usuário pode acessar os botões diretamente no quadro relacionado, evitando que o mesmo se sinta perdido, procurando as funcionalidades.

Falta de ajuda: Caso algum usuário leigo precisasse ajuda quanto às funcionalidades da interface, ele deveria pedir a alguém que soubesse, pois o sistema não contava com nenhum tipo de ajuda referente à interface, somente às operações. Este problema foi solucionado com a adição de vídeos de tutorial. Ao acessar o sistema o usuário pode assistir ao vídeo acessando um link na própria página do projeto.

Esses são alguns dos problemas que foram possíveis de solucionar. A Figura 1 ilustra o estado atual da interface gráfica do PATequation com estes problemas já resolvidos.

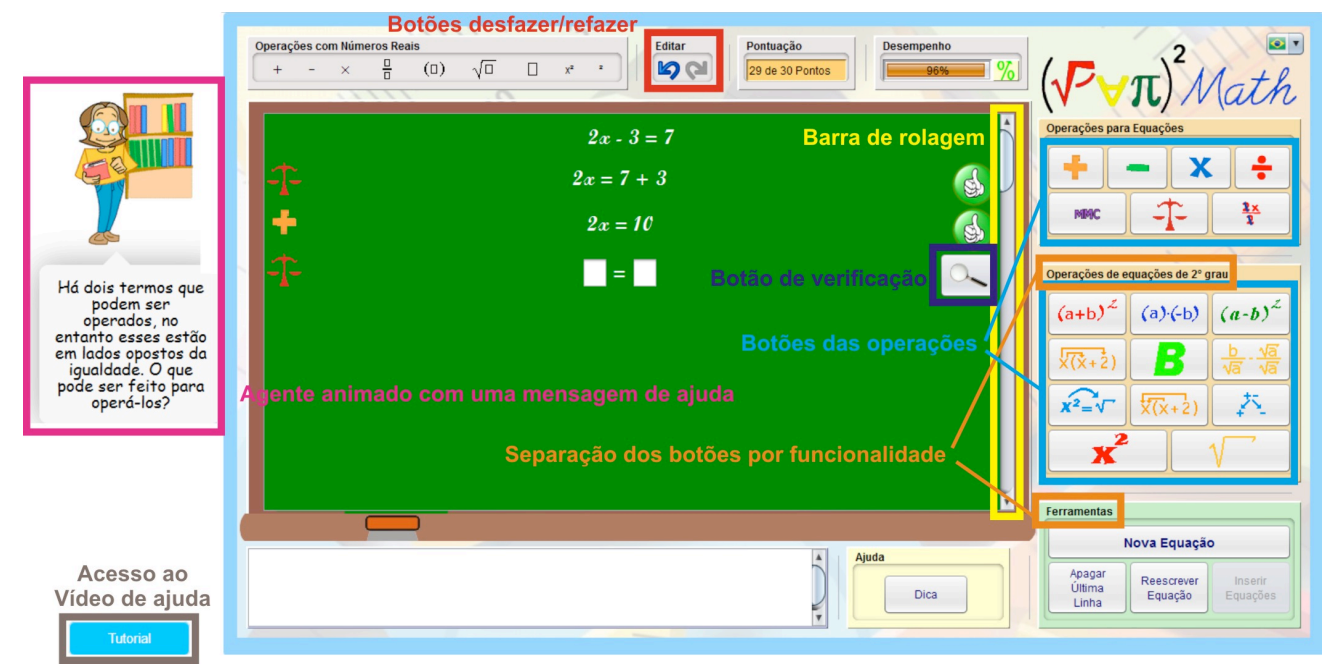

Figura 1: Interface gráfica do PATequation com alguns dos problemas resolvidos. 
Os itens citados nos problemas da lista de inconformidades estão destacados na Figura 1 para melhor compreensão. Esta é a última versão da interface, que por sua vez, já está adequada com aquelas inconformidades. Portanto, esta foi a versão da interface submetida à avaliação heurística de usabilidade com especialistas.

\section{Avaliação Heurística no PATequation}

Baseada nas heurísticas de usabilidade, a avaliação heurística, proposta por (Nielsen, 1994), visa identificar e evitar os possíveis erros encontrados na interface antes que testes de usabilidade, com usuários, sejam realizados. Este método de avaliação, geralmente, é realizado por avaliadores experientes, ou seja, pessoas que já estejam familiarizadas com o sistema.

O principal objetivo é encontrar os erros mais aparentes no sistema. Dessa forma, eles podem ser resolvidos antes da avaliação heurística com usuários, evitando assim, que a grande maioria dos usuários venha a documentar erros já conhecidos. Normalmente, de 3 a 5 avaliadores especialistas participam desta avaliação, documentando os erros e inconformidades encontrados (Nielsen, 1994).

A interface do PATequation foi submetida a uma avaliação heurística de usabilidade. A equipe de avaliadores foi constituída por 6 integrantes, sendo eles 2 programadores do próprio projeto (de outros módulos do PAT2Math que não o PATequation) e outros 4 programadores sem ligação ao projeto. Todos os avaliadores possuem experiência com desenvolvimento web. É interessante salientar que uma segunda motivação para uma avaliação heurística com especialistas foi devido ao fato de que os usuários potenciais do sistema são muito jovens (geralmente, jovens de 12 a 14 anos), podendo não entender o real objetivo da avaliação.

O objetivo desta avaliação era identificar os principais problemas de usabilidade na interface do PATequation. Para isso, cada avaliador resolveu 3 equações utilizando a interface, sendo que, após a utilização, todos responderam um questionário. Este questionário era formado por 12 questões objetivas e 2 discursivas. As objetivas englobavam todas as heurísticas de usabilidade, enquanto que nas discursivas, o avaliador deveria informar sobre alguma dificuldade ao utilizar o sistema e dar sugestões de melhoria.

Nas questões objetivas foi utilizada a escala Likert (Likert, 1932) para medir o grau de concordância dos avaliadores com cada afirmação do questionário. Os avaliadores deveriam dar uma nota de 1 a 5 para cada questão, as quais representam: 1 discordo totalmente, 2 discordo parcialmente, 3 indiferente, 4 concordo parcialmente e 5 concordo totalmente.

A Tabela 1 ilustra as 12 questões e quantos avaliadores deram cada nota em cada questão. Esses valores foram obtidos dos questionários de cada um dos 6 avaliadores.

Tabela 1: Amostra dos dados coletados através de um questionário em uma avaliação heurística da interface do PATequation.

\begin{tabular}{|c|l|r|r|r|r|r|}
\hline № & \multicolumn{1}{|c|}{ Questão } & $\mathbf{1}$ & $\mathbf{2}$ & $\mathbf{3}$ & $\mathbf{4}$ & $\mathbf{5}$ \\
\hline $\mathbf{1}$ & $\begin{array}{l}\text { As respostas que o sistema emite quando realizo alguma } \\
\text { ação são suficientes para compreender o resultado da } \\
\text { ação no sistema. }\end{array}$ & 0 & 0 & 2 & 1 & 3 \\
\hline
\end{tabular}




\begin{tabular}{|c|c|c|c|c|c|c|}
\hline 2 & $\begin{array}{l}\text { As mensagens que o sistema emite utilizam palavras de } \\
\text { fácil compreensão. }\end{array}$ & 0 & 0 & 1 & 3 & 2 \\
\hline 3 & $\begin{array}{l}\text { A posição dos textos e componentes da interface se } \\
\text { encontra em um local adequado e fácil de encontrar. }\end{array}$ & 0 & 1 & 1 & 4 & 0 \\
\hline 4 & $\begin{array}{l}\text { A interface me permite desistir e cancelar uma ação já } \\
\text { iniciada. }\end{array}$ & 0 & 0 & 2 & 3 & 1 \\
\hline 5 & $\begin{array}{l}\text { A interface é consistente, utilizando componentes, } \\
\text { mensagens e ações semelhantes e padronizadas. }\end{array}$ & 0 & 0 & 1 & 3 & 2 \\
\hline 6 & $\begin{array}{l}\text { A interface de usuário não possui elementos } \\
\text { desnecessários ao contexto da ferramenta. }\end{array}$ & 0 & 2 & 1 & 2 & 1 \\
\hline 7 & $\begin{array}{l}\text { Os comandos e ações possíveis estão disponíveis na } \\
\text { interface de forma que não precise relembrá-los ou } \\
\text { perder muito tempo para encontrá-los. }\end{array}$ & 0 & 2 & 2 & 2 & 0 \\
\hline 8 & $\begin{array}{l}\text { As mensagens de erro emitidas pelo sistema são de fácil } \\
\text { compreensão e ajudam a compreender o problema. }\end{array}$ & 0 & 0 & 3 & 3 & 0 \\
\hline 9 & $\begin{array}{l}\text { As mensagens de erros emitidas pelo sistema informam } \\
\text { como resolver o problema. }\end{array}$ & 0 & 0 & 3 & 2 & 1 \\
\hline 10 & $\begin{array}{l}\text { O contraste entre as letras e as cores permite fácil leitura } \\
\text { sem dificultar a navegação. }\end{array}$ & 0 & 0 & 1 & 4 & 1 \\
\hline 11 & $\begin{array}{l}\text { O sistema possui um sistema de ajuda facilmente } \\
\text { acessível. }\end{array}$ & 0 & 0 & 1 & 3 & 2 \\
\hline 12 & $\begin{array}{l}\text { O sistema possui atalhos suficientes para agilizar a } \\
\text { interação do usuário. }\end{array}$ & 0 & 2 & 1 & 3 & 0 \\
\hline
\end{tabular}

Ao analisar os dados ilustrados na Tabela 1, pode-se notar que embora várias melhorias tenham sido feitas, conforme as sugestões e reclamações dos alunos, ainda há correções substanciais a serem realizadas no sistema. Na questão 6, por exemplo, apenas 3 dos 6 avaliadores afirmaram que a interface não contém informações desnecessárias, o não é considerado bom. Outro exemplo é na questão 7, na qual apenas 2 avaliadores afirmaram que não precisam relembrar das suas ações na interface. Este é um problema preocupante em softwares educacionais, pois ao armazenar informações sobre a interface gráfica, o usuário acaba sobrecarregando sua memória de trabalho com informações inúteis quanto à resolução de equações. Isso é conhecido como carga cognitiva (cognitive load). Pesquisas como a de (Reis et al., 2012) vem mostrando que o aumento da carga cognitiva pode prejudicar o processo de aprendizagem. Na questão 12 , os avaliadores sentiram a falta de atalhos para agilizar o processo, o que deixa os usuários mais experientes entediados, podendo, portanto, abandonar o sistema.

As 2 questões discursivas obtiveram respostas bem distintas. Nessas questões cada avaliador deu sua opinião e relatou os problemas encontrados durante a utilização do sistema. Alguns dos problemas encontrados pelos avaliadores estão detalhados na lista a seguir:

Resolução da tela: A interface gráfica do PATequation é um applet, desenvolvido em Java, que executa dentro de uma página HTML. Porém, o tamanho desta interface é fixo, não permitindo que usuários com um monitor de baixa resolução utilizem o sistema perfeitamente. Além disto, o applet executa dentro de uma página HTML, o que não permite que outros componentes gráficos o sobreponham. Por isto o agente animado não pode se locomover dentro da página. Usuários com monitor de 
baixa resolução devem ficar movendo a barra de rolagem do navegador para poder ver toda a interface e o agente animado.

Selecionar termos: A obrigatoriedade em fazer que os usuários selecionem os termos em algumas operações apenas (e em outras não) foi bastante comentado. Os usuários ficam indecisos em quando devem selecionar os termos ou apenas clicar no botão da operação. Isso ocorre em operações de adição, subtração, multiplicação e divisão, nas quais os usuários devem selecionar os termos a serem operados, ao contrário da operação inversa, por exemplo, que basta clicar no botão que representa esta operação.

Excesso de botões e ícones não intuitivos: Ao implementar a interface, para cada operação disponível foi adicionado um botão que representava essa operação através de um ícone. Como há muitos botões e ícones para representar as operações, suas imagens e aparências não são intuitivas aos usuários.

Obrigatoriedade de indicar a operação: O processo de indicar qual a operação deve ser utilizada em cada passo na resolução de uma equação é obrigatório. Para cada operação há um botão, e o usuário deve escolher o botão correspondente a operação que ele deve realizar. Porém esse processo de indicar a operação se mostra incômodo para os usuários, além de não se mostrar pedagogicamente muito efetivo. Isso porque não há uma vantagem pedagógica do usuário saber o nome da operação a ser realizada. $\mathrm{O}$ importante é que ele saiba quando aplicar uma operação e como aplica-la. Isso pode ser avaliado sem a presença dos botões de operações.

Esses foram alguns dos problemas encontrados pelos avaliadores do sistema. Porém, esses problemas ultrapassam os limites das tecnologias utilizadas. Esse é o caso do agente animado não poder ficar na frente da interface gráfica, o que faz com que o mesmo fique imóvel em um canto da página. Para solucionar todos os esses problemas, foi proposto o desenvolvimento de uma nova interface gráfica, utilizando uma tecnologia mais atual e com mais recursos gráficos, o HTML 5. A Figura 2 ilustra a nova interface gráfica proposta para o PATequation.

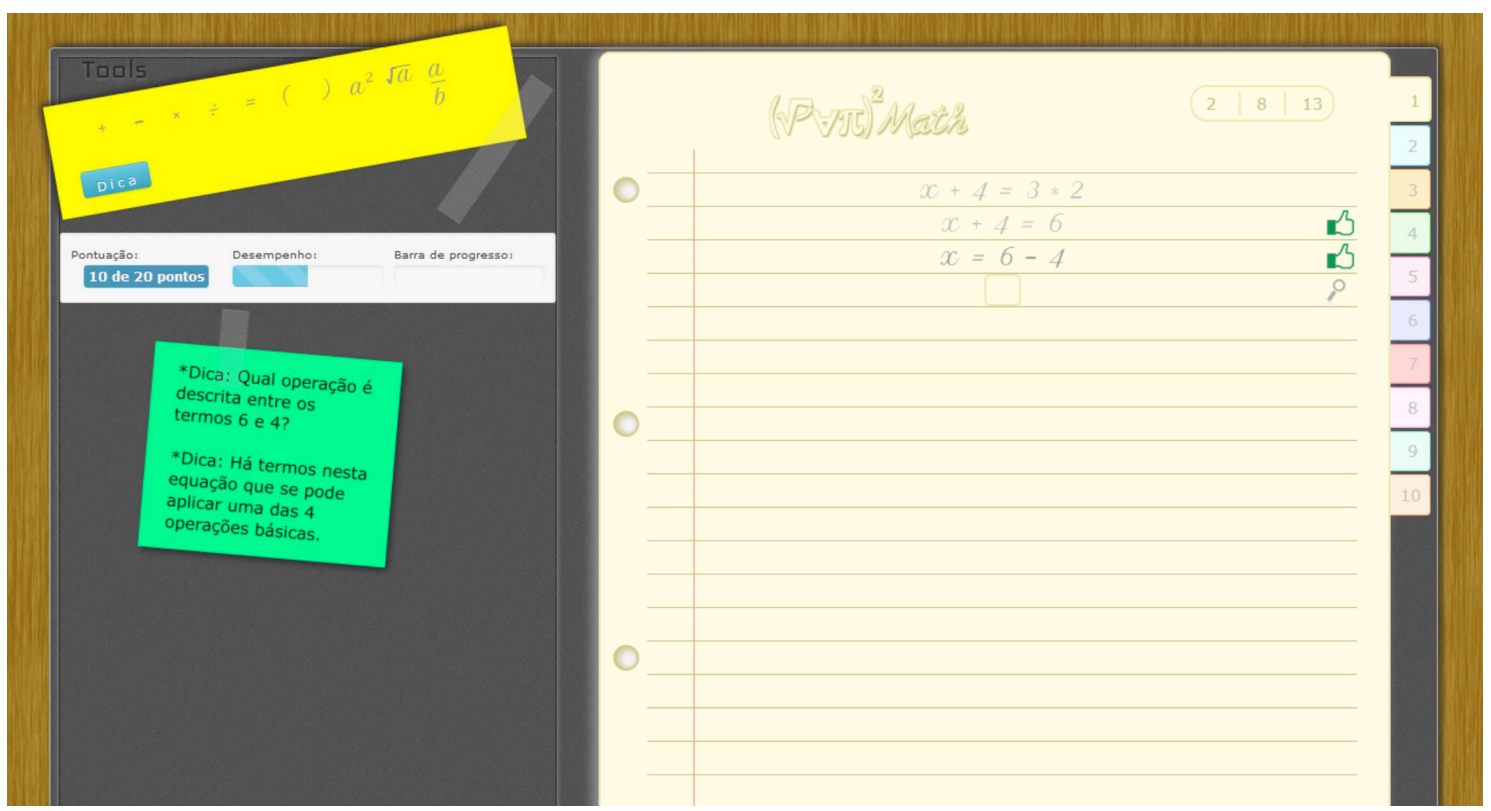

Figura 2: Nova interface gráfica do PATequation. 
A nova interface gráfica já está funcionando para equações de primeiro e segundo grau, porém ainda está em fase de testes e de integração com o ambiente web do projeto PAT2Math. No seu desenvolvimento está sendo utilizado HTML 5, CSS 3 e JavaScript. Essas tecnologias suportam todos os problemas de incompatibilidade encontrados com a antiga interface. $\mathrm{O}$ usuário não precisa mais selecionar termos e escolher a operação que está utilizando. Ao iniciar a resolução de uma equação, a interface habilita apenas um campo de texto no meio da linha, no qual ele pode digitar o que desejar como solução. Após digitar a solução daquele determinado passo, ele pode clicar no botão de verificação ou apertar a tecla "Enter" e a interface lhe fornece um feedback. Não é mais necessário informar a operação utilizada ou selecionar itens da equação.

As equações já resolvidas, que antes ficavam inacessíveis ao usuário, agora ficam acessíveis através de um sistema de abas do lado direito da página. Por exemplo, o usuário pode voltar a uma equação já resolvida e relembrar alguma operação utilizada. O sistema exibe igualmente a data em que a equação foi resolvida pelo aluno. Outra melhoria implementada é em relação às dicas. Na versão anterior, as mensagens de ajuda eram exibidas apenas nos balões de fala do agente. Essas mensagens eram exibidas apenas por um breve período de tempo, enquanto o agente estivesse falando. Dessa forma, não era possível reler as mensagens. Agora a interface mantém as mensagens em uma janela de lembrete, no qual o usuário pode revê-las inúmeras vezes. Além dessas melhorias, também foi implementada uma barra de progresso, cujo objetivo é informar ao usuário quantas equações de um determinado tópico ele já resolveu e quantas ainda faltam pra resolver.

O agente animado utiliza a mesma tecnologia empregada no desenvolvimento da nova interface. Portanto, agora ele poderá se locomover por toda a página sem restrições. Essa liberdade proporcionou alguns benefícios. Por exemplo, ao fornecer uma explicação ou uma mensagem de ajuda, o agente também poderá apontar para partes específicas de uma equação. Essa integração entre o agente animado e a interface gráfica encontra-se em desenvolvimento.

\section{Conclusão}

A interface gráfica do PATequation é o principal meio de comunicação do aluno com o STI. É através dela que o usuário pode resolver as equações e obter feedback instantâneo a cada interação. Com o objetivo de avaliar a usabilidade desta interface, para que seja mais eficiente possível, ela foi submetida a uma avaliação de usabilidade. Esta avaliação segue as heurísticas de usabilidade e pode ser chamada de avaliação heurística. Através desta avaliação heurística foi possível identificar vários problemas de usabilidade na interface do PATequation. Esta avaliação foi importante, pois mostrou que mesmo melhorando a interface, ela ainda teria inconformidades com as heurísticas de usabilidade. Para solucionar esses problemas e inconformidades foi proposta uma nova interface gráfica utilizando uma tecnologia mais atual.

A utilização de métodos de avaliação de usabilidade em interfaces gráficas é o que torna a interação do usuário com o sistema mais acessível e menos frustrante. Quanto maior a confiança e o entendimento do usuário com o sistema, maior será sua confiabilidade, sendo que em STIs, mais o aluno poderá aprender com o sistema. 
Como trabalhos futuros, será finalizada a fase de testes e a integração com o ambiente web do projeto. Após a conclusão, essa nova interface será submetida a uma avaliação heurística para depois ser avaliada com usuários.

\section{Agradecimentos}

O presente trabalho foi realizado com o apoio dos seguintes órgãos de fomento à pesquisa: CAPES, CNPq e FAPERGS.

\section{Referências Bibliográficas}

Jacko, J. A. and Stephanidis, C. Human-computer Interaction: Theory and Practice. Part I. Lawrence Erlbaum Associates, Incorporated. 2003.

Jaques, P. A., Lehmann, M. and Pesty, S0. Evaluating the affective tactics of an emotional pedagogical agent. In Proceedings of the 2009 ACM Symposium on Applied Computing. ACM. 2009.

Jaques, P. A., Seffrin, H., Rubi, G., et al. Rule-based Expert Systems to support step-bystep guidance in Algebraic Problem Solving: the case of the Tutor PAT2Math. Expert Systems with Applications, v. 40, n. 14, pag. 5456-5465. 2013.

Jaques, P. A., Vicari, R., Pesty, S. and Martin, J. C. Evaluating a cognitive-based affective student model. In Proceedings of the 4th international conference on Affective computing and intelligent interaction - Volume Part I. Springer-Verlag. 2011.

Koedinger, K. R., Anderson, J. R., Hadley, W. H. and Mark, M. A. Intelligent tutoring goes to school in the big city. International Journal of Artificial Intelligence in Education (IJAIED), v. 8, p. 30-43. 1997.

Likert, R. A technique for the measurement of attitudes. Archives of psychology. 1932.

Nielsen, J. Usability engineering. Elsevier. 1994.

Psotka, J., Massey, L. D. and Mutter, S. A. Intelligent Tutoring Systems: Lessons Learned. LAWRENCE ERLBAUM ASSOC Incorporated. 1988.

Reis, H. M., Borges, S. S., Durelli, V. H., et al. Towards Reducing Cognitive Load and Enhancing Usability through a Reduced Graphical User Interface for a Dynamic Geometry System: An Experimental Study. In Multimedia (ISM), 2012 IEEE International Symposium on. IEEE. 2012.

Seffrin, H. M., Rubi, G., Carlotto, T., Mello, G. and Jaques, P. A. Um resolvedor de equações algébricas como ferramenta de apoio à sala de aula no ensino de equações algébricas. In WORKSHOP SOBRE INFORMÁTICA NA ESCOLA. 2009.

Seffrin, H., Rubi, G., Da Cruz, B. and Jaques, P. Resolvendo equações algébricas no STI PAT2Math. In Anais do Simpósio Brasileiro de Informática na Educação. 2010.

Seffrin, H., Rubi, G., Morais, F., et al. Dicas inteligentes no Sistema Tutor Inteligente PAT2Math. In Anais do Simpósio Brasileiro de Informática na Educação. 2012.

Shneiderman, B. and Ben, S. Designing The User Interface: Strategies for Effective Human-Computer Interaction, 4/e (New Edition). Pearson Education India. 2003.

Woolf, B. P. Building intelligent interactive tutors: Student-centered strategies for revolutionizing e-learning. Morgan Kaufmann. 2008. 